\title{
Excitation of the Chandler Wobble
}

\author{
N. S. Sidorenkov \\ Hydrometeorological Centre of the Russian Federation, Bol'shoi \\ Predtechenskii per. 9-13, Moscow, Russia. \\ e-mail: sidor@glasnet.ru
}

\begin{abstract}
The redistribution of air and water masses between the $\mathrm{Pa}$ cific and Indian oceans during the El Niño/Southern oscillation (ENSO) changes the components of the Earth's inertia tensor and shifts the position of the pole of the Earth's rotation. The spectrum of the ENSO has components with periods of about $6,3.6,2.8$, and 2.4 years. These periods are all the multiples of the Chandler period $T=1.2 \mathrm{yr}$. and the principal period of nutation $18.6 \mathrm{yr}$. A nonlinear model for the Chandler polar motion has been constructed based on this empirical fact. In this model, the ENSO excites the Chandler polar motion by acting on the Earth at the frequencies of combinative resonance. At the same time, the Chandler polar motion induces a polar tide in the atmosphere and the World Ocean, which orders the ENSO. As a result, the dominant components in the noise spectrum of the ENSO are those with the periods indicated above.
\end{abstract}

\section{Introduction}

The motion of the geographical poles has two main harmonic components: oscillations with the Chandler period and oscillations with the period of a year. The yearly oscillation is due to the seasonal redistribution of air and water masses between the continents and oceans. It is thought that the motion of the poles with the Chandler period $(1.2 \mathrm{yr}$.) is the free oscillation of the Earth relative to its fixed rotation axis or the Earth's free nutation. The Earth is not a perfectly elastic body. The kinetic energy of its free nutation should therefore dissipate into thermal energy. No damping of the Chandler polar motion (CPM) is observed, however. Consequently, there must be some mechanism that excites and maintains this motion. The problem of the excitation mechanism for the Chandler polar motion has been discussed in literature for roughly 100 years. The most comprehensive reviews are given in (Munk \& McDonald 1960, Yatskiv et al. 1976, Lambeck 1980).

Of possible processes that could excite the CPM, those most frequently considered are meteorological and seismic. Most estimates of the influence of meteorological effects show that they are inadequate. Opinions are quite contradictory about the effect of earthquakes on the excitation of the Chandler polar motion. In addition, the simple estimates of the possible effect of earthquakes (see, for example, Stacey (1969)) show that it is negligibly small. 
In 1992, we suggested (Sidorenkov 1992a) that the CPM is excited by the El Niño Southern oscillation (ENSO). Here, we investigate the effect of the ENSO on the Chandler polar motion in a more detailed way.

\section{Original Data}

A great variety of indices have been suggested to describe the ENSO. The Southern oscillation index (SOI), defined as follows, is used for operational monitoring purposes. First, the difference of the normalized pressure anomalies at the Tahiti and Darwin stations is calculated:

$$
\delta_{g m}=\left[\frac{P_{g m}-\overline{P_{m}}}{\varepsilon}\right]_{T a}-\left[\frac{P_{g m}-\overline{P_{m}}}{\varepsilon}\right]_{D a},
$$

where $P_{g m}$ is the observed atmospheric pressure over the month $m$ in the year $g$, $\overline{P_{m}}$ is the mean pressure (norm) for the month $m$ over many years, and $\varepsilon$ is the standard deviation calculated for all pressure anomalies from 1951 to 1980 . The norms, are determined from the mean monthly data over the period 1951-1980. Next, the values of the index

$$
\dot{S} O I=\frac{\delta_{g m}}{\nu},
$$

are calculated where $\nu$ is the standard deviation of all differences from 1951 to 1980. The continuous and homogeneous series of average monthly values of the SOI from 1935 to the present time is well-known (Ropelewski \& Jones 1987, Sidorenkov 1992b). Observations at the Tahiti and Darwin stations were also conducted before 1935 , but they have been inaccessible for a long time, since they have been scattered among the archives of various institutions. Tedious searches by a number of researchers made it possible to reconstruct the continuous series of atmospheric pressure data at these stations from 1866 to 1934 (Ropelewski \& Jones 1987, Allan et al. 1991). As a result, we have at our disposal the continuous average monthly record of the SOI from 1866 to the present time.

Wright (1989) calculated the mean quarterly ENSO indices $W$ starting from 1851 using atmospheric pressure data for several (up to eight) stations. To eliminate inhomogeneities and fill gaps in the pressure series, Wright used ENSO indices based on the ocean surface temperature, rainfall, and air temperature in the central and eastern parts of the Pacific ocean. He later abandoned the $W$ index and proposed the $D T$ index - the difference in the pressure anomalies of the Darwin and Tahiti stations. It was straightforward to calculate the $D T$ index for dates starting from 1935. Using the synchronous $D T$ and $W$ series for 1935-1974, Wright found a regression equation relating $D T$ to $W$; he then used the $W$ indices for the period 1851-1934 to calculate the $D T$ indices for this period. We adopted the published Wright $D T$ indices (Wright 1989), and added calculated values for the indices for the last 11 years. As a result, we obtained a continuous series of the Wright $D T$ index over the last 145 years. 


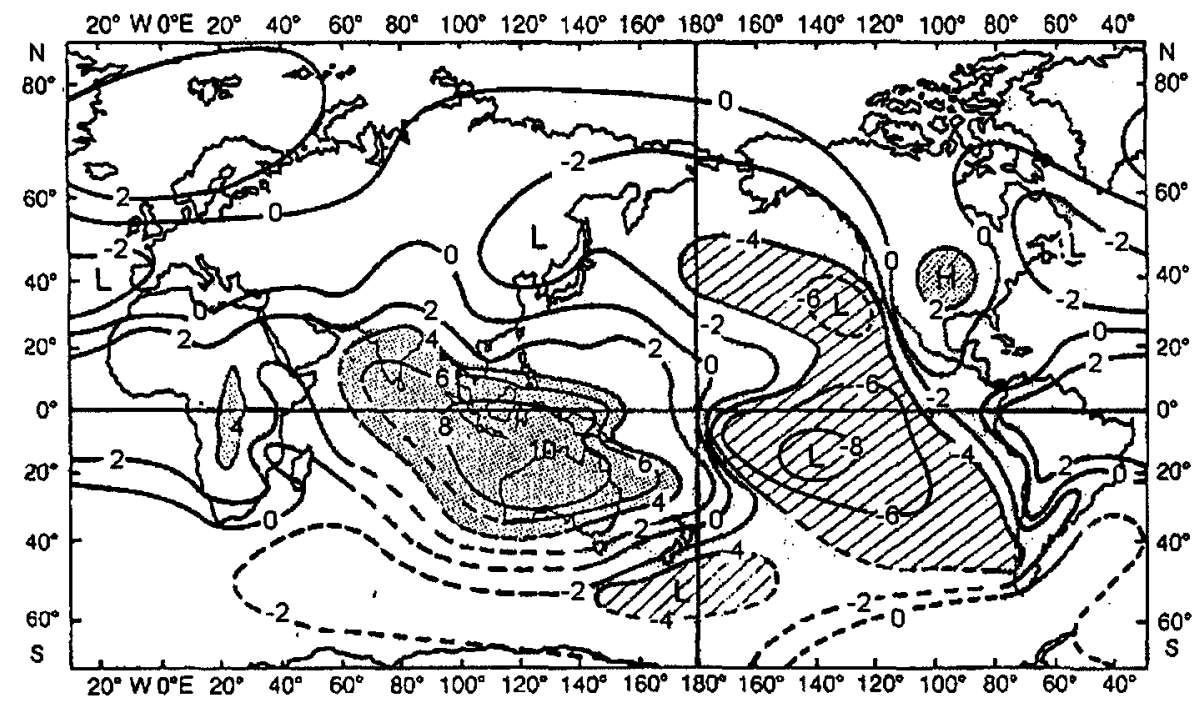

Figure 1. The correlation $(\times 10)$ of annual mean sea-level pressures with those for Darwin, Australia (after K. Trenberth (1976)). Note the high negative correlations over Tahiti - showing the basis for the choice of the Tahiti-Darwin pressure difference as a Southern Oscillation Index (SOI).

\section{Spectral Analysis}

We performed the spectral analysis of all the available series of ENSO indices: 580 quarterly $D T$ values and 1563 monthly $S O I$ values. The two series were centered and normalized (i.e. each series has a zero mean and unit dispersion), so that it would be straightforward to compare the estimates of their spectra. In the calculations of the spectra of the $D T$ and $S O I$ indices, we used 290 and 781 correlation function values, respectively. We used Parzen windows with widths of 145 values ( $D T$ index) and 360 values $(S O I)$ to smooth the spectra.

The spectral density reaches the maximum in the range of periods exceeding one year. At shorter periods, the spectral density oscillates between zero and unity. For this reason the spectral density curves for periods longer than one year are shown in Figure 2.

Curves 1 and 2 represent the spectra estimates for the $S O I$ and $D T$ indices, respectively. It is evident that the $S O I$ spectrum is dominated by a component with the period of $3.6 \mathrm{yr}$., while the $D T$ spectrum is dominated by a component with the period of $5.8 \mathrm{yr}$. In both curves, there are spectral density peaks at the periods of about $6,3.6,2.8$, and 2.4 years. There is also a peak near $12 \mathrm{yr}$. One striking feature of these periods is that, they all are multiples of the Chandler period of $1.2 \mathrm{yr}$. and the principal period of nutation $18.6 \mathrm{yr}$. 


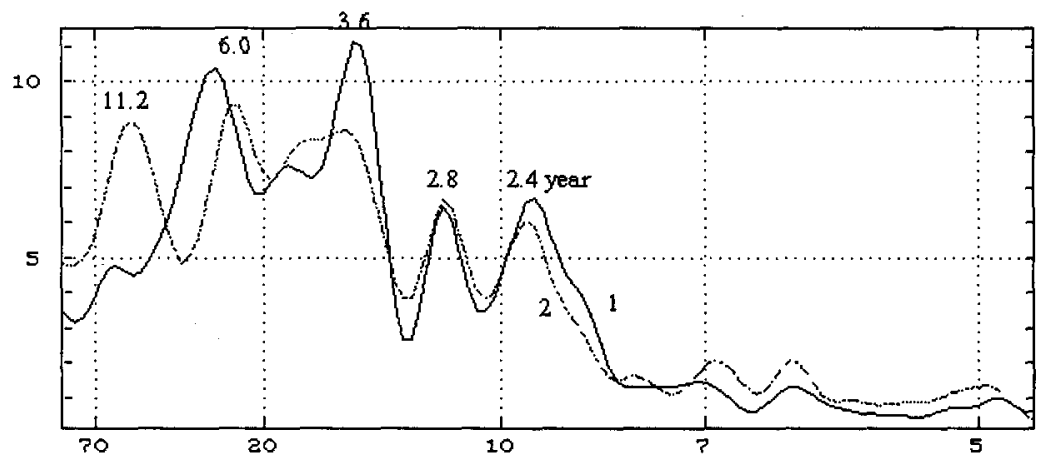

Figure 2. The power spectra of the $S O I(1)$ and $D T(2)$ indices. The periods (in quarters of a year) are given on the horizontal axis and the spectral density is indicated on the vertical axis.

\section{The Model of Nonlinear Oscillations}

During the ENSO, air and water masses are redistributed between the western and eastern hemispheres. The exchange is particularly intense between the southern subtropical part of the Pacific Ocean and the eastern part of the Indian Ocean, where the amplitude of the atmospheric pressure oscillations reaches 3 mbar (Fig. 1) (Trenberth 1976, Sidorenkov 1992b). The mass redistribution gives rise to changes in the Earth's inertia tensor, which is described by the expression

$$
I_{i j}=\iint_{V} \int \rho\left(x_{l}^{2} \delta_{i k}-x_{i} x_{k}\right) d v
$$

in a geocentric Cartesian coordinate system. The variation of the centrifugal moments of inertia excites motion of the Earth's geographical poles according to the equation (Munk \& McDonald 1960, Lambeck 1980):

$$
\frac{i}{\bar{\sigma}} \frac{d \bar{m}}{d t}+\bar{m}=\bar{\Psi}
$$

Here, $\bar{\sigma}=\sigma+i \beta, \sigma=\frac{2 \pi}{T}$ is the frequency of the free polar motion; $T=1.2 \mathrm{yr}$. is the Chandler period; $\beta$ is the damping coefficient and, $\bar{m}=m_{1}+i m_{2}$; where $m_{1}$ and $m_{2}$ are the direction cosines of the instantaneous rotational angular velocity vector of the Earth. $\bar{\Psi}=\chi_{1}+i \chi_{2}$, where $\chi_{1}$ and $\chi_{2}$ are the direction cosines of the vector excitation function

$$
\bar{\Psi} \approx \frac{n_{13}+i n_{23}}{C_{m}-A_{m}} \approx-\frac{R^{2}}{2 g\left(C_{m}-A_{m}\right)} \iint_{S} P \sin 2 \theta e^{i \lambda} d s,
$$

where $n_{13}$ and $n_{23}$ are the centrifugal moments of inertia of the atmosphere, $C_{m}$ and $A_{m}$ are the principle moments of the inertia of the Earth's mantle, $R$ is the Earth's radius, $g$ is the acceleration of gravity, $\rho$ is density, $P$ is the atmospheric pressure, $\theta$ is the complement of the latitude; $\lambda$ is the longitude, and $S$ is the 
surface of the Earth. Since the excitation function $\bar{\Psi}$ depends on the distribution of the atmospheric pressure $P$ at the Earth's surface, its spectrum could be the same as the $S O I$ spectrum, i.e., it could have components with the periods 6 , 3.6 , and 2.4 years, the multiples of the period $T$. This is our first assumption about the function $\bar{\Psi}$.

At present Salstein \& Rosen (1997) has calculated the components of function from 1958 to 1999 . We have performed the spectral analysis of these 41-year series of the components. The results are presented in the Table 1 . They confirm the presence of the cycle of $6,3.6$, and 2.4 years, - the multiples of the periods $T$.

Table 1. The dominant cycles of the ENSO and components of the EAAM.

\begin{tabular}{|c|c|}
\hline \hline Components & Period, year \\
\hline$S O I$ & $3.6 ; 6 ; 2.8 ; 2.4$ \\
$D T$ & $5.8 ; 3.6 ; 2.8 ; 12 ; 2.4$ \\
$\chi_{1}^{w}$ & $2.7 ; 3.7 ; 1.86 ; 5.9 ; 2.4$ \\
$\chi_{2}^{w}$ & $2.7 ; 3.1 ; 2.1 ; 1.8$ \\
$\chi_{3}^{w}$ & $2.4 ; 3.7 ; 5.1$ \\
$\chi_{1}^{p i b}$ & $2.6 ; 3.7 ; 4.6 ; 1.7$ \\
$\chi_{2}^{p i b}$ & $2.4 ; 2.7 ; 5.9$ \\
$\chi_{3}^{p i b}$ & $5.1 ; 4.1 ; 2.3 ; 2.7$ \\
$18.6 y r$. & $6.2 ; 4.7 ; 3.7 ; 3.1 ; 2.7 ; 2.3 ; 2.1$ \\
\hline
\end{tabular}

The Chandler polar motion generates a polar tide in the atmosphere and the ocean. In the World Ocean, for example, the static polar tide has the form (Munk \& McDonald 1960)

$$
\zeta=-\frac{1+k-h}{g} \frac{\Omega^{2} R^{2}}{2} \sin 2 \theta\left(m_{1} \cos \lambda+m_{2} \sin \lambda\right),
$$

where $k$ and $h$ are the Love numbers and $\Omega$ is the modulus of the angular velocity vector for the Earth's rotation. The typical amplitude of the static polar tide is $0.5 \mathrm{~cm}$. However, the analysis of level gauge data indicates the presence of a resonance peak in the spectral density at the period $T$ (Munk \& McDonald 1960). The polar tide in the atmosphere is described by the same expression, but with different amplitude. According to empirical data, the amplitude of the atmospheric polar tide is about 1 mbar. We want to emphasize that this amplitude is the function of the polar deviation $\bar{m}$. It is clear from equation (5) that the amplitude $A$ of the oscillations of the excitation function $\bar{\Psi}$ should also be a function of $\bar{m}$. It is most likely that $A_{k}=\mu_{k} \bar{m}^{\alpha_{k}}$, where $\alpha_{k} \neq 1$. This is our second assumption about the function $\bar{\Psi}$.

Using these results and assumption, we can write the function $\bar{\Psi}$ to the first approximation by the sum of $N$ harmonics

$$
\bar{\Psi}=\sum_{k=1}^{N} \mu_{k} \bar{m}^{\alpha_{k}} \exp \left[\omega_{k} i\left(t-t_{0 k}\right)\right] \approx \sum_{k=1}^{N} \mu_{k} \bar{m}^{\alpha_{k}} \exp \left(\omega_{k} i t\right)
$$




$$
\approx \bar{m}^{\alpha} \sum_{k=1}^{N} \mu_{k} \exp \left(\omega_{k} i t\right),
$$

where $k$ is the harmonic number, $\omega_{k}=\frac{\sigma}{n_{k}}, n_{k}=2,3,5,10$. Let $\bar{m}=\bar{m}_{0}$ at $t=0$. Then, the solution to equation (4) with the function $\bar{\Psi}$ in the form given by (7) is

$$
\begin{gathered}
\bar{m}(t)=\left\{\begin{array}{c}
\bar{m}_{0}^{1-\alpha} \exp [(1-\alpha) \bar{\sigma} i t]-i \bar{\sigma}(1-\alpha) \exp [(1-\alpha) \bar{\sigma} i t] \\
\quad \times \int_{0}^{t} \exp [(\alpha-1) \bar{\sigma} i \tau]\left(\sum_{k=1}^{N} \mu_{k} \exp \left(\omega_{k} i \tau\right)\right) d \tau
\end{array}\right\}^{\frac{1}{1-\alpha}}= \\
\left\{\bar{m}_{0}^{1-\alpha} \exp [(1-\alpha) \bar{\sigma} i t]+\sum_{k=1}^{N} \frac{\mu_{k}(1-\alpha) \bar{\sigma}}{\left[(1-\alpha) \bar{\sigma}-\omega_{k}\right]}\left[\exp \left(i \omega_{k} t\right)-\exp [(1-\alpha) i \bar{\sigma} t]\right\}^{\frac{1}{1-\alpha}}\right. \\
\approx \bar{m}_{0} e^{i \bar{\sigma} t}+\sum_{k=1}^{N}\left(\mu_{k} \omega_{k}\right)^{n_{k}} t^{n_{k}} \exp \left[i\left(\sigma t+\frac{3 n_{k} \pi}{2}\right)\right]
\end{gathered}
$$

This result shows that for $(1-\alpha)=\frac{\omega_{k}}{\sigma}=\frac{1}{n_{k}}$, the amplitude of the Chandler polar motion grows proportional to $t^{n_{k}}$ without bound, i.e., there is a combinative resonance - the phenomenon characteristic of nonlinear oscillations.

\section{Discussion}

The ENSO changes the components of the atmospheric inertia tensor and consequently excites motions of the Earth's pole. The figure, structure, and physical properties of the Earth are such that the period of the free oscillations of the Earth's pole is $1.2 \mathrm{yr}$. In addition to this Chandler motion, there is the forced motion of the pole with the period of $1 \mathrm{yr}$. The sum of these two motions gives rise to beats, which cause the radius $m$ of the polar trajectory to vary from its maximum to its minimum value with the period of approximately six years.

The motion of the pole at the same time induces a polar tide in the atmosphere and the world ocean. The amplitude of this tide depends on the value of $|\bar{m}|$, i.e. there are nonlinear oscillations. The "controlling" effect of the polar tide leads to the modest ordering of the ENSO, and to the appearance of cyclic components with periods that are the multiples of the Chandler period in its spectrum. As a result, a combinative resonance arises, in which even low-power effects can excite intense polar motion. It is important to emphasize that excitation of the Chandler polar motion occurs mainly at the periods $6,4.8,3.6$, 2.4 year. In this way, the ENSO can continuously maintain the Chandler polar motion.

Time variations in the intensity of the ENSO lead to instabilities in the Chandler polar motion excitation process, and thus to variations in its characteristics (amplitude, phase, damping decrement, etc.). For example, the significant damping of the Chandler polar motion (a several-fold decrease in its amplitude, lengthening of its period, and a change in its phase) was observed in 1925-1945 
(Munk \& McDonald 1960, Yatskiv et al. 1976, Lambeck 1980). At that same time, there were also significant anomalies in the recurrence interval for the warm phases of the ENSO (Sidorenkov 1992b). Phases with $S O I<0$ appeared rarely, and from 1930 to 1940 there were no long intervals with $S O I<0$ at all. The maximum amplitudes of the Chandler polar motion were observed approximately during 1910 and 1955. For 10-15 years before these epochs, the warm phases were especially long and intense. The most important fact is that they were the multiples of the Chandler period. These examples demonstrate the connection between the Chandler polar motion and the ENSO. Thus the El Niño/Southern Oscillation and the Chandler polar motion are both the component parts of the same global process - year-to-year variations in the Atmosphere-Ocean-Earth System.

We believe that the negative results concerning the role of meteorological processes in exciting the Chandler polar motion have been obtained in part due to the very sparse network of meteorological observations in the southern hemisphere (the location of the ordered oscillations of air masses between the Pacific and Indian oceans that drive the Earth). In addition, temporal series over several decades are needed to detect weak components with periods of $6,3.6$, and 2.4 years in the spectrum of the effective atmospheric angular momentum function. The series of atmospheric angular momentum data has currently been accumulated for only 41 years. The temporal series of the monthly atmospheric moments of inertia for 1873-1950 calculated by Hassan (1961) was limited to the barometric observations on the continents. This series does not include the measurements of atmospheric pressure in the Pacific and the Indian oceans and, therefore, its analysis cannot be expected to give reliable results.

Acknowledgments. We thank A.N. Filatov for his help in this work. This work was supported by International Astronomical Union, The Cariplo Foundation for scientific research and the Russian Foundation for Basic Research.

\section{References}

Allan, R.J., Nicholls, N., Jones, P.D., and Butterworth, I.J., 1991, J. Climate, $4,743$.

Hassan, E., 1961, Meteorological Monographs, 24, 1.

Lambeck, K., 1980, The Earth's Variable Rotation: Geophysical Causes and Consequences, Cambridge, Cambridge Univ. Press.

Munk, W.H. \& McDonald, G.J.R., 1960, The Rotation of the Earth: A Geophysical Discussion, Cambridge, Cambridge Univ. Press.

Ropelewski, C.F. \& Jones, P.D., 1987, Mon. Wea. Rev., 115, 2161.

Salstein, D.A. \& Rosen, R.D., 1997, Preprints, 7th Conf. on Climate Variations, American Meteorological Society, Boston, MA, 344-348.

Sidorenkov, N.S., 1992a, A Zh, 69, 905.

Sidorenkov, N.S., 1992b, Trudy Gidrometsentra SSSR, No. 316, 31 (in Russian).

Stacey, F.D., 1969, Physics of the Earth, New York, Wiley.

Trenberth, K. E., 1976, Quart. J. Roy. Meteorol. Soc., 102, 639.

Wright, P.B., 1989, Int. J. Climatol., 9, 33. 
Yatskiv, Ya.S., Mironov, N.T., Korsuń, A.A., and Taradii, V.K., 1976, Itogi Nauki Tekh., Ser. Astron., Moscow: VINITI, 12, part 1 (in Russian). 